\title{
THERMODYNAMIC PROPERTIES OF NANOTUBES: ZONE-FOLDING APPROACH
}

\author{
R.A. Evarestov, A.V. Bandura, and V.V. Porsev \\ Quantum Chemistry Department, Saint-Petersburg State University, 7/9 Universitetskaya nab., 199034 St. Petersburg, \\ Russia \\ E-mail: r.evarestov@spbu.ru
}

Received 3 October 2015; revised 19 January 2016; accepted 21 June 2016

\begin{abstract}
A zone-folding approach is applied for the estimation of phonon contributions to the thermodynamic properties of carbon nanotubes and nanotubes based on transition metal oxides $\left(\mathrm{TiO}_{2}, \mathrm{~V}_{2} \mathrm{O}_{5}\right)$ and sulfides $\left(\mathrm{TiS}_{2}, \mathrm{ZrS}_{2}\right)$ with different morphology and various chiralities. The results obtained are compared with those from the direct calculation of the thermodynamic properties of nanotubes in the harmonic approximation. All calculations have been made using the PBE0 hybrid exchange-correlation functional. It is found that the zone-folding approach allows a sufficiently accurate estimation of phonon contributions to internal energy and heat capacity and shows worse but acceptable results for Helmholtz free energy and entropy.
\end{abstract}

Keywords: carbon nanotubes, metal oxide nanotubes, metal disulfide nanotubes, thermodynamic properties of nanotubes, zone folding

PACS: 61.43.Bn, 61.46.Np, 65.80.+n

\section{Introduction}

The theoretical construction of nanotube (NT) models is usually based on the rolling up of the diperiodic nanolayers cut form the bulk crystals [1]. In some sense this procedure is equivalent to imposing of cyclic boundary conditions on the layer supercell in the direction which becomes the tube circumference. This circumstance is utilized in the so-called zone-folding (ZF) approach [2-3] by means of cutting the electron or phonon dispersion in the $2 \mathrm{D}$ reciprocal space of the layer used for NT rolling up. In such a way the properties of single-wall nanotubes (SWNTs) with sufficiently large diameters can be estimated from the results of the corresponding layer calculation. The ZF approximation was widely used for the electron and phonon state calculations of the single-wall carbon nanotubes (SWCNTs) rolled up from the graphene layer [2-7]. Few zone-folding calculations are also known for the hexagonal boron nitride [8, 9].

The thermodynamic properties of SWNT can be obtained in the harmonic approximation by the summation of vibrational contributions over all the phonon modes of the unit cell and over $k$-values in the onedimensional Brillouin zone (BZ) of SWNT. One can hope that the role of the curvature effects in this case is not as large as it is for separate phonon states. One can expect that the sum over low-frequency modes (which are essentially different in the nanolayer and the corresponding NT) forms a relatively small part in the integral sums used for the calculations of thermodynamic properties. Nevertheless, this point requires special study to understand the ZF approach possibilities to reproduce correctly the difference in the thermodynamic properties both for SWNTs of different chiralities and the same composition and for SWNTs with different compositions. These issues are addressed in our current study. Particularly, in the present paper we have verified the ZF approximation on SWCNTs, single-walled $\mathrm{TiO}_{2}^{-}$, $\mathrm{TiS}_{2}^{-}$, and $\mathrm{ZrS}_{2}$-based NTs with hexagonal morphology and on $\mathrm{V}_{2} \mathrm{O}_{5}$-based NTs with rectangular morphology. To the best of our knowledge, the ZF approach has never been applied to the computation of NT thermodynamic properties.

\section{Zone-folding approach}

To specify the SWNT with chirality $(n, m)$ we should define two vectors: the rolling vector $\mathbf{C}_{\mathrm{h}}=n \mathbf{a}_{1}+m \mathbf{a}_{2}$ and the translation vector $\mathbf{T}=t_{1} \mathbf{a}_{1}+t_{2} \mathbf{a}_{2}$, where $\mathbf{a}_{1}$ and 
$\mathbf{a}_{2}$ are the primitive translations of the $2 \mathrm{D}$ direct lattice. The SWNT translation vector $\mathbf{T}\left(t_{1}\right.$ and $t_{2}$ are supposed to have no common divisor except unity) is orthogonal to the vector $\mathbf{C}_{\mathrm{h}}:\left(\mathbf{T} \cdot \mathbf{C}_{\mathrm{h}}\right)=0$. The unrolled unit cell of the nanotube is exactly a rectangular supercell built on the vectors $\mathbf{C}_{h}$ and $\mathbf{T}$. It is periodically repeated in the direction of the translation vector $\mathbf{T}$ and has a finite nanoscale size in the direction of the chiral vector $\mathbf{C}_{h}$.

The transformation from the primitive translation vectors $\left[\mathbf{a}_{1}, \mathbf{a}_{2}\right]$ to the supercell translation vectors $\left[\mathbf{C}_{h}, \mathbf{T}\right]$ can be expressed in the matrix form:

$$
\left(\begin{array}{c}
\mathbf{C}_{\mathrm{h}} \\
\mathbf{T}
\end{array}\right)=\mathbf{Q}\left(\begin{array}{c}
\mathbf{a}_{1} \\
\mathbf{a}_{2}
\end{array}\right), \quad \mathbf{Q}=\left(\begin{array}{cc}
n & m \\
t_{1} & t_{2}
\end{array}\right), \quad \operatorname{det}(\mathbf{Q})=N .
$$

Here $N=n t_{2}-m t_{1}$ is the number of layer unit cells in the rectangular supercell formed by vectors $C_{h}$ and $T$.

Taking into account the well-known theoretical relations [10], the transformation in the reciprocal space of the layer corresponding to (1) in the direct space of the layer can be written as

$$
\left(\begin{array}{l}
\mathbf{K}_{1} \\
\mathbf{K}_{2}
\end{array}\right)=\left(\mathbf{Q}^{\mathrm{T}}\right)^{-1}\left(\begin{array}{l}
\mathbf{b}_{1} \\
\mathbf{b}_{2}
\end{array}\right)=\frac{1}{N}\left(\begin{array}{rr}
t_{2} & -t_{1} \\
-m & n
\end{array}\right)\left(\begin{array}{l}
\mathbf{b}_{1} \\
\mathbf{b}_{2}
\end{array}\right),
$$

where $\left[\mathbf{b}_{1}, \mathbf{b}_{2}\right]$ are the primitive vectors of the reciprocal lattice, and $\left[\mathbf{K}_{1}, \mathbf{K}_{2}\right]$ are the new primitive translations in the reciprocal space of the layer, corresponding to the supercell translation vectors $\mathbf{C}_{h}$ and T. Eq. (2) gives

$$
\begin{aligned}
& \mathbf{K}_{1}=\left(t_{2} \mathbf{b}_{1}-t_{1} \mathbf{b}_{2}\right) / N, \\
& \mathbf{K}_{2}=\left(-m \mathbf{b}_{1}+n \mathbf{b}_{2}\right) / N .
\end{aligned}
$$

Any vector $\mathbf{k}$ in the reciprocal space can be expressed either in the basis [b] or the basis [K]:

$$
\begin{aligned}
& \mathbf{k}=\left(l_{1}, l_{2}\right)[\mathbf{b}]=l_{1} \mathbf{b}_{1}+l_{2} \mathbf{b}_{2}, \\
& \mathbf{k}=\left(k_{1}, k_{2}\right)[\mathbf{K}]=k_{1} \mathbf{K}_{1}+k_{2} \mathbf{K}_{2} .
\end{aligned}
$$

It is well known that vector components are transformed with the inverse matrix of the basis set transformation, so

$$
\left(k_{1}, k_{2}\right)=\left(l_{1}, l_{2}\right) \mathbf{Q}^{\mathrm{T}}
$$

or

$$
k_{1}=n l_{1}+m l_{2}, k_{2}=t_{1} l_{1}+t_{2} l_{2} .
$$

The layer rolling up means introduction of the cyclic boundary condition in the circumferential direction so that the SWNT (unrolled) wave function $\psi_{\mathrm{L}}$ satisfies the Bloch theorem

$$
\psi_{\mathrm{L}}\left(\mathbf{r}+\mathbf{C}_{\mathrm{h}}\right)=\mathrm{e}^{\mathrm{ikCh}} \psi_{\mathrm{L}}(\mathbf{r})=\psi_{\mathrm{L}}(\mathbf{r}) .
$$

It means that for any $\mathbf{k}=k_{1} \mathbf{K}_{1}+k_{2} \mathbf{K}_{2}$ in the reciprocal space of the layer the following condition has to be satisfied with the integer $q$ :

$$
\left(\mathbf{k} \cdot \mathbf{C}_{\mathrm{h}}\right)=k_{1}\left(\mathbf{K}_{1} \cdot \mathbf{C}_{\mathrm{h}}\right)=2 \pi k_{1}=2 \pi\left(n l_{1}+m l_{2}\right)=2 \pi q .(7)
$$

So, the unrolled reciprocal space of the nanotube is quantized along the $\mathbf{K}_{1}$ direction and is continuous along the $\mathbf{K}_{2}$ direction. Using Eq. (3a) for the vector $\mathbf{K}_{1}$, one can see that $N \mathrm{~K}_{1}$ is a translation vector of the original $2 \mathrm{D}$ reciprocal space of the layer. This is the shortest translation vector in the given direction, since the integer numbers $t_{1}$ and $t_{2}$ do not have a common divisor by definition. None of the $N$ vectors $q \mathbf{K}_{1}$ $(q=0,1, \ldots N-1)$ is a layer's reciprocal lattice vector.

Finally, we can conclude that the allowed $\mathbf{k}=l_{1} \mathbf{b}_{1}+l_{2} \mathbf{b}_{2}$ are constrained by the equation $k_{1}=q$. It means that

$$
n l_{1}+m l_{2}=q
$$

or

$$
\begin{array}{cc}
l_{2}=-(n / m) l_{1}+q / m & \text { if } m \neq 0, \\
l_{1}=q / n & \text { if } m=0,
\end{array}
$$

and therefore $\mathbf{k}$ belongs to the lines in the reciprocal space of the layer (the so-called "cutting lines", that are parallel to the tube axis and the reciprocal vector $\mathbf{K}_{2}$ ). The position of each line is defined by a concrete value of $q=0,1, \ldots N-1$. Condition (8) does not affect the $k_{2}$ values, $-1 / 2<k_{2} \leq 1 / 2$, which correspond to the first Brillouin zone in the reciprocal space of onedimensional nanotube.

Each of $N$ quantized states with $\mathbf{k}=q \mathbf{K}_{1}$ gives rise to a line segment of the length $K_{2}=\left|\mathbf{K}_{2}\right|$ along the direction $\mathbf{K}_{2}$ in the unrolled reciprocal space of the nanotube. These $N$ line segments defined by the wave vectors $\mathbf{K}_{1}$ and $\mathbf{K}_{2}$ represent the cutting lines in the unrolled reciprocal space of the nanotube [2]. The length and orientation of each cutting line in the reciprocal space are given by the wave vector $\mathbf{K}_{2}$, and the separation between two adjacent cutting lines is given by the wave vector $\mathbf{K}_{1}$. This representation of cutting lines is called [2] the fully $\mathbf{K}_{1}$-extended representation. In Fig. 1 we illustrate the supercell formation in the direct lattice $(N=28)$ and the fully extended representation of cutting lines in the reciprocal lattice for the chiral $(8,2)$ SWCNT with hexagonal morphology. It should be noted that relations (1)-(8) are valid for any type of the $2 \mathrm{D}$ lattice except an oblique one for which the orthogonality condition cannot be satisfied.

In the phonon frequency calculation we use a direct (frozen-phonon) method [11, 12,. To realize 
(a)

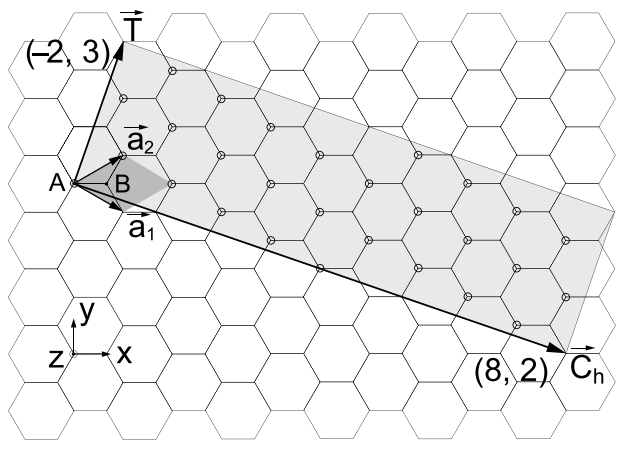

(b)

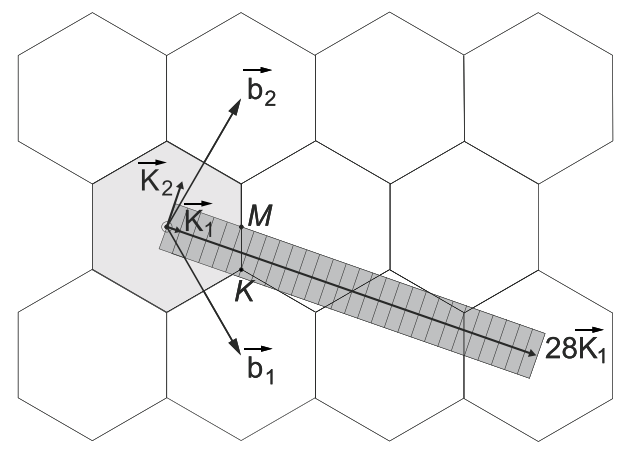

Fig. 1. The supercell construction in the direct (a) and reciprocal (b) 2D hexagonal lattice for the chiral $(8,2)$ SWCNT with $N=28.28$ cutting lines are shown in part (b).

the zone-folding approach, we need to construct the supercell which provides the folded modes corresponding to the cutting lines. This is possible with using the vectors $\mathrm{C}_{\mathrm{h}}$ and $M \mathrm{~T}$, where $M$ is some integer resulting in accounting of $M$ equidistance points in each cutting line. Upon the cell expansion, the $\Gamma$-point of the reduced $\mathrm{BZ}$ accommodates $N M \mathbf{k}$-points from the original BZ of the layer. Such an approach, however, requires an additional study of the convergence of the calculated properties while increasing $M$.

\section{Calculation of layer and nanotube thermodynamic properties}

In the harmonic approximation, the vibrational part $E_{\mathrm{ph}}(T)$ of the internal energy per unit cell of the slab or SWNT includes the zero point vibrational energy (ZPVE) $E_{v 0}$ and the temperature dependent vibrational contribution $E_{\mathrm{v}}(T)$ [13]:

$$
\begin{aligned}
& E_{\mathrm{v} 0}=\frac{1}{N_{\mathbf{k}}} \sum_{\mathbf{k}}^{N_{\mathrm{k}}} \sum_{i=1}^{N_{\mathrm{v}}} \frac{1}{2} h \mathrm{v}_{i}(\mathbf{k}), \\
& E_{\mathrm{ph}}(T)=E_{v 0}+E_{\mathrm{v}}(T), \\
& E_{\mathrm{v}}(T)=\frac{1}{N_{\mathbf{k}}} \sum_{\mathbf{k}}^{N_{\mathrm{k}}} \sum_{i=1}^{N_{\mathrm{v}}} \frac{h v_{i}(\mathbf{k})}{\exp \left[\left(h / k_{\mathrm{B}}\right) v_{i}(\mathbf{k}) / T\right]-1} .
\end{aligned}
$$

Here $k_{\mathrm{B}}$ is the Boltzmann constant, $h$ is the Planck constant, and $i$ numbers are the phonon modes with frequencies $v_{i}$. The summation over $i$ in (9) and (10) is made over $N_{v}$ branches per slab or SWNT unit cell. The summation over $\mathbf{k}$ in (9) and (10) is made over $N$ values in the $\mathbf{K}_{1}$ direction for the slab and over $M$ values in the $\mathbf{K}_{2}$ direction both for the layer and SWNT, i. e. $N_{\mathbf{k}}=M$ in the case of the SNWT and $N_{\mathbf{k}}=N M$ in the case of the layer used in $\mathrm{ZF}$.

The phonon contribution $A_{\mathrm{ph}}(T)$ to the Helmholtz free energy at temperature $T$ is calculated by the wellknown [13] equation:

$$
\begin{aligned}
& A_{\mathrm{ph}}(T)=E_{\mathrm{v} 0}+A_{\mathrm{v}}(T), \\
& A_{\mathrm{v}}(T)=\frac{1}{N_{\mathbf{k}}} \sum_{\mathbf{k}}^{N_{\mathrm{k}}} \sum_{i=1}^{N_{\mathrm{v}}} k_{\mathrm{B}} T \ln \left\{1-\exp \left[-\left(h / k_{\mathrm{B}}\right) \mathrm{v}_{i}(\mathbf{k}) / T\right]\right\} .
\end{aligned}
$$

If Helmholtz and internal energies are known, the entropy can be calculated as $S(T)=(E(T)-A(T)) / T$. The harmonic contributions to the heat capacity at constant volume can be expressed in the following form [13]:

$$
\begin{aligned}
& C_{V}(T)=\frac{1}{N_{\mathbf{k}}} \sum_{\mathbf{k}}^{N_{\mathbf{k}}} \sum_{i=1}^{N_{v}} C_{V i}(T, \mathbf{k}) \\
& C_{V i}(T, \mathbf{k})=k_{\mathrm{B}}\left(\frac{h}{k_{\mathrm{B}}} \frac{v_{i}(\mathbf{k})}{T}\right)^{2} \frac{\exp \left[\left(h / k_{\mathrm{B}}\right) v_{i}(\mathbf{k}) / T\right]}{\left\{\exp \left[\left(h / k_{\mathrm{B}}\right) v_{i}(\mathbf{k}) / T\right]-1\right\}^{2}} .
\end{aligned}
$$

\section{Computational details}

The PBE0 exchange-correlation functional [14 has been used in our calculations based on the hybrid Hartree-Fock-density functional theory. All computations have been performed using the CRYSTAL14 computer code within the linear combination of atomic orbitals (LCAO) [15, 16]. For C, O and V atoms we have used a consistent portable basis set of the triple-zeta valence with the polarization quality (pob-TZVP) [17]. To describe the interaction between the core and valence electrons in $\mathrm{S}, \mathrm{Ti}$, and $\mathrm{Zr}$ atoms we use CRENBL [18-20] relativistic effective core pseudopotentials (ECPs). The corresponding AO exponents and coefficients are given elsewhere [21].

Kohn-Sham equations are solved iteratively to selfconsistency within $3 \times 10^{-9} \mathrm{eV}$. The Monkhorst-Pack scheme [22] with the $144 \times 144 \mathbf{k}$-point mesh has been applied for the graphene layer BZ sampling. This extremely dense mesh is needed to provide the sufficient accuracy for semimetallic graphene. The BZ of semiconducting metal oxide and metal sulfide hexagonal layers was sampled using the regular $16 \times 16$ mesh. 
The $\mathrm{BZ}$ of the $\mathrm{V}_{2} \mathrm{O}_{5}$ layer with rectangular morhology was sampled using the $12 \times 4$ mesh. The lattice parameters and fractional positions of atoms were optimized until the forces on each atom were less than $1.5 \mathrm{meV} \cdot \AA^{-1}$. The harmonic vibrational frequencies have been obtained by the frozen-phonon method [11, 12]. Two $k$-points $(0$ and $1 / 2)$ in $1 D$ BZ are sufficient to estimate the temperature dependence of the thermodynamic functions as it is shown below. The corresponding frequencies have been determined from the numerical second-order derivatives of the ground state energy using the analytical first-order derivatives. The finite-displacement central-difference method is used for this purpose in the CRYSTAL14 code [12, 23].

The calculation method used provides a sufficiently good description of the structure of the systems under consideration. Indeed, the calculated lattice constants, bond distances and atomization energies agree well with the experimental bulk properties of all the considered systems. Thus, the lattice constants of bulk phases are reproduced within 1-2\% except the value of $c$ in graphite which is underestimated by $4 \%$. The agreement between the obtained graphene frequencies and the experimental values is also satisfactory.

\section{Results and discussion}

\subsection{Graphene-based nanotubes}

The symmetry of SWCNTs was analyzed in many publications, using the graphene layer folding approach [24-26. The symmetry of the graphene layer is described by hexagonal layer group $80(\mathrm{P6} / \mathrm{mmm})$ being a $2 \mathrm{D}$ subgroup of graphite symmetry space group $194\left(\mathrm{PG}_{3} / \mathrm{mmc}\right)$. As follows from Ref. [27], after the rolling procedure the symmetry of the achiral $(n, 0)$ and $(n, n)$ SWCNTs is described by the line group $L(2 n) n / m c m$ belonging to family 13 (point symmetry $\left.D_{2 n h}\right)$. The symmetry of the chiral $(n, m)$ SWCNT is described by the line groups $L(N)_{s} 22$ or $L(N)_{S} 2$ (depending on the simultaneous parity of $N$ and $S$ ) belonging to family 5 (point symmetry $D_{N}$ ).

Due to a relatively simple structure, the SWCNTs allow the possibility of a detailed study of the dependence of thermodynamic functions on the chirality, diameter $(D)$, and NT period $(|\mathbf{T}|)$. For two different nanotubes having close diameters, it is easier to model a nanotube with a shorter translation vector $\mathbf{T}$ due to a smaller number of atoms in a $1 \mathrm{D}$ unit cell. By ordering of 2D lattice translation vectors by length we can obtain the smallest supercells for SWNTs rolling up. The lowest $N$ value for the hexagonal lattice is two as the smallest rectangular supercell consists of two hexagons. Two sets of translation vectors correspond to $N=2$. The first one is formed by the three smallest translation vectors (up to sign) of the length $a\left(\mathbf{a}_{1},-\mathbf{a}_{2},-\mathbf{a}_{1}+\mathbf{a}_{2}\right)$. Using the orthogonality conditions [2, 24, 27] in the case of the hexagonal lattice with the angle $\gamma=60^{\circ}$ between the primitive translation vectors

$$
t_{1} / t_{2}=-(2 m+n) /(2 n+m)
$$

and

$$
n / m=-\left(2 t_{2}+t_{1}\right) /\left(2 t_{1}+t_{2}\right),
$$

we can obtain three equivalent armchair nanotubes with $N=2$ and primitive (up to sign) chirality indexes: $(1,-2),(-2,1)$, and $(1,1)$. The $n$-time increasing of these chirality indexes gives $N=2 n$. The second set consists of the three translation vectors of the length $a \sqrt{3},\left(\mathbf{a}_{1}+\mathbf{a}_{2}, \mathbf{a}_{1}-2 \mathbf{a}_{2},-2 \mathbf{a}_{1}+\mathbf{a}_{2}\right)$, that can be used to obtain three equivalent zig-zag nanotubes with the chirality indexes $(1,-1),(-1,0)$ and $(0,1)$. After the $n$-time increasing we have again $N=2 n$. At last, $N=14 n$ for the translation vectors of the next length $a \sqrt{7},\left(-\mathbf{a}_{1}-2 \mathbf{a}_{2},-2 \mathbf{a}_{1}+3 \mathbf{a}_{2}, 3 \mathbf{a}_{1}-\mathbf{a}_{2}\right)$, giving the chirality vectors $(-5 n, 4 n),(4 n, n)$ and $(n,-5 n)$ of the equivalent chiral nanotubes. It should be noted that $N=14 n$ can also correspond to SWNTs with the translation vector of the length $a \sqrt{21}$ giving among the others the chirality $(2 n, n)$.

Here we consider the following examples of each mentioned chirality type: armchair $(6,6)$, zig-zag $(10,0)$, and chiral $(8,2)$ and $(4,2)$. The structure of the first three relevant NTs is shown in Fig. 2. The ZF approach for $(8,2)$ SWCNT is illustrated in Fig. 1 .

At the first stage of our SWCNT study we explored the convergence of the calculated quantities with the number $M$ of $k$-points in the 1D NT BZ. We found that two points $(0,1 / 2)$ in $1 \mathrm{D}$ BZ are sufficient for the estimation of phonon contributions to the NT thermodynamic functions of the SWCNTs of any translation period. This number of $k$-points provides the NT internal energy within an accuracy of $0.02 \mathrm{~kJ} \mathrm{~mol}^{-1}$ (per mole of atoms), the NT free energy within an accuracy of $0.05 \mathrm{~kJ} \mathrm{~mol}^{-1}$, and the NT entropy and heat capacity within an accuracy of 0.15 and $0.10 \mathrm{~J} \mathrm{~mol}^{-1} \mathrm{~K}^{-1}$, respectively, in the temperature interval from 0 to $600 \mathrm{~K}$.

In Fig. 3. (a) we compare the temperature dependences of $A_{v}(T)$ calculated with $M=1,2$, and 3 for the carbon SWNT $(6,6)$ and the corresponding graphene layer used in the ZF approach. A similar comparison made in Fig. 3(b) for $\operatorname{SWCNT}(8,2)$ shows 


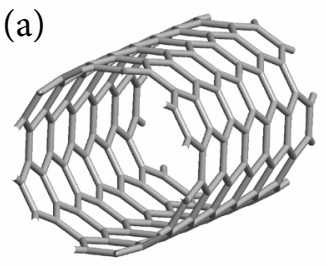

(b)
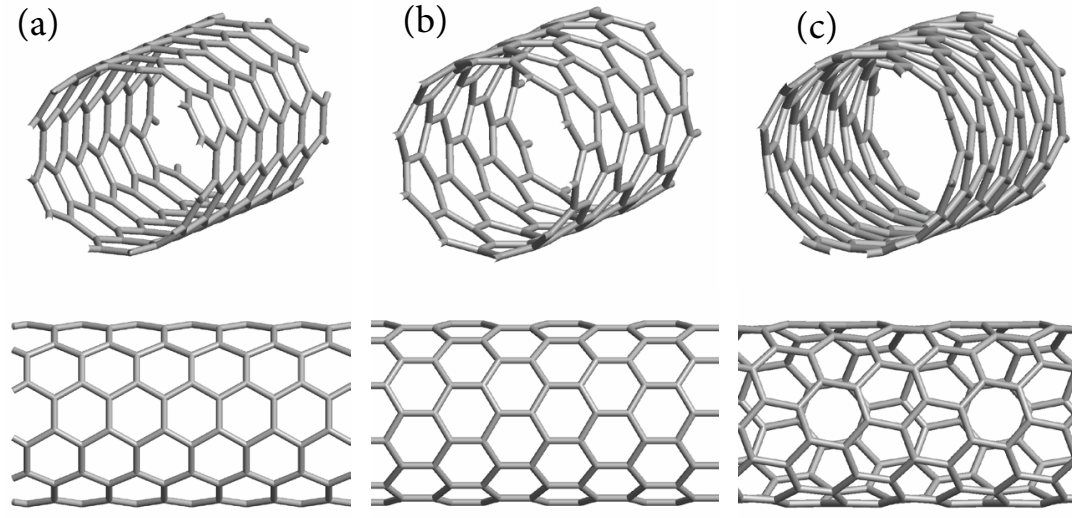

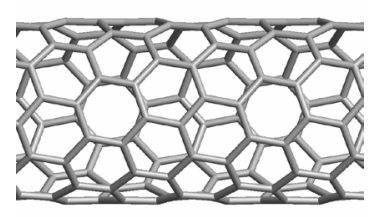

Fig. 2. Carbon SWNTs with diameter $D$ of about $8 \AA$ : armchair $(6,6)(a)$, zig-zag $(10,0)(b)$, and chiral $(8,2)$ (c). A perspective view on the top and a side view on the bottom. that $M=1$ is sufficient for the NTs with a translational period greater than $10 \AA$. In all of the subsequent calculations we use $M=2$.

To verify the ZF approach we performed both the direct and ZF calculations of the thermodynamic

(a)

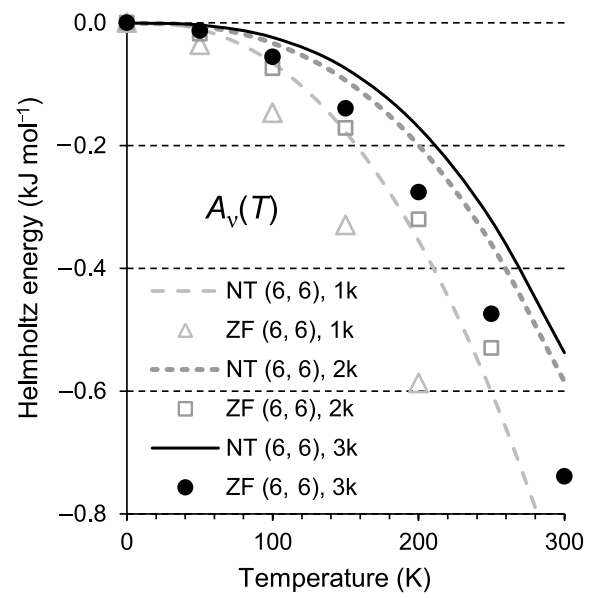

(b)

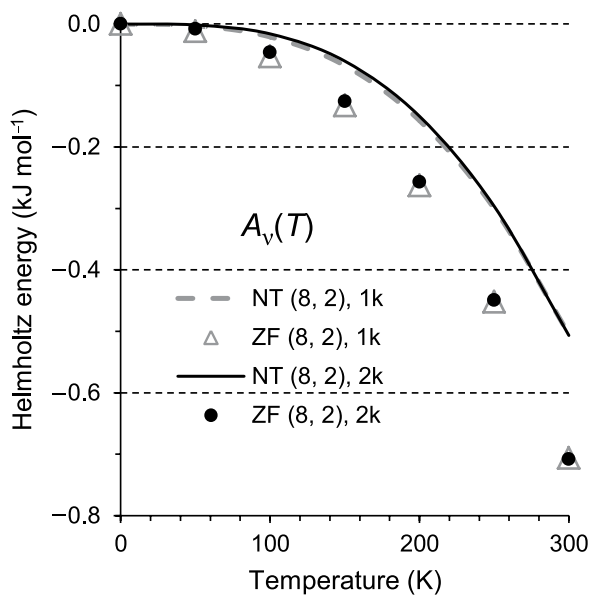

Fig. 3. Dependence of thermal contribution to Helmholtz energy on the number $M$ of $k$-points in the $1 \mathrm{D} \mathrm{BZ}$ summation for SWCNTs: (a) achiral $(6,6) \mathrm{NT}$ (green online) with diameter $D=8.14 \AA$ and period $|\mathrm{T}|=4.89 \AA$; (b) chiral $(8,2) \mathrm{NT}$ (blue online) with diameter $D=7.19 \AA$ and period $|\mathbf{T}|=12.93 \AA$. functions for several carbon nanotubes and corresponding ZF layer models. We found that the agreement between the two approaches was good in the case of zero point vibration energy, it was satisfactory in the case of internal energy and heat capacity, but appeared to be worse in the case of free energy and entropy (see Fig. 4). These results can be explained by the fact that the weights of the low-frequency modes in ZPVE are evidently small (see Eq. (9)), and they contribute to the free energy (and entropy) to a larger extent than to the internal energy (and heat capacity). The latter is obvious from Eqs. (10-12).

To demonstrate this, in Fig. 4 we compare the temperature dependence of the thermal contributions in the case of achiral SWCNTs $(12,12)$ and $(12,0)$ calculated directly and using the ZF approach. The screw axis $24_{12}$ describes the rototranslational symmetry of those NTs. It is clearly seen that the agreement between the results of $\mathrm{ZF}$ and NT calculations is better for the internal energy and heat capacity than for the free energy and entropy. Moreover, this agreement improves with increasing the NT diameter.

A similar comparison of chiral SWCNTs $(8,2)$ and $(4,2)$ is given in Fig. 5. These NTs have both different diameters and different translation periods; the first one has the screw axis $28_{18}$ and the second one has $28_{22}$. However, their thermodynamic properties calculated directly are practically the same. This fact can be understood by taking into account that the unit cells of these tubes have been obtained by rolling up of the same layer supercell, which corresponds to both $(8,2)$ and $(4,2)$ nanotubes if $M=2$ in the ZF model.

At $300 \mathrm{~K}$ the average distance between the curves of NT and ZF data in Figs. $3-5$ is about $0.1 \mathrm{~kJ} \mathrm{~mol}^{-1}$ for the internal energy $E_{v}(\mathrm{~T})$ and $0.2 \mathrm{~kJ} \mathrm{~mol}^{-1}$ for the Helmholtz free energy $A_{v}(\mathrm{~T})$. However, the relative position of the curves for different chiralities is the same for NT and ZF calculations. 

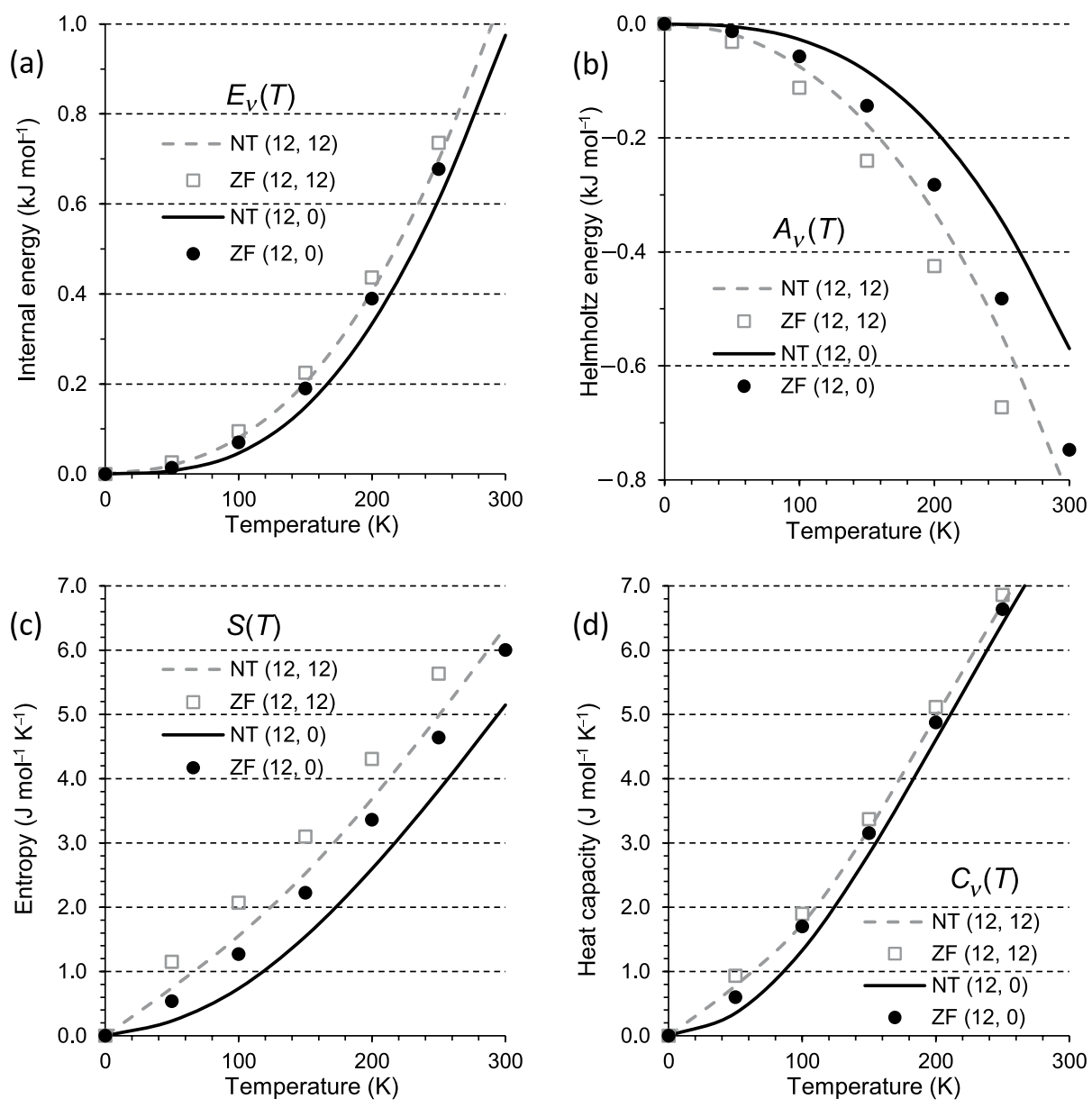

Fig. 4. Temperature dependence of thermal contributions to internal energy (a), Helmholtz energy (b), entropy (c), and heat capacity (d) for achiral SWCNTs. NT $(12,0)$ : $D=9.38 \AA,|\mathrm{T}|=8.47 \AA$, $E_{v 0}=16.88 \mathrm{~kJ} \mathrm{~mol}^{-1}, E_{v 0}^{\mathrm{ZF}}=$ $16.74 \mathrm{~kJ} \mathrm{~mol}^{-1}$; NT $(12,12$, blue online): $D=16.20 \AA,|\mathbf{T}|=$ $4.89 \AA E_{0}=16.86 \mathrm{~kJ} \mathrm{~mol}^{-1}$, $E_{0}^{\mathrm{ZF}}=16.82 \mathrm{~kJ} \mathrm{~mol}^{-1}$.
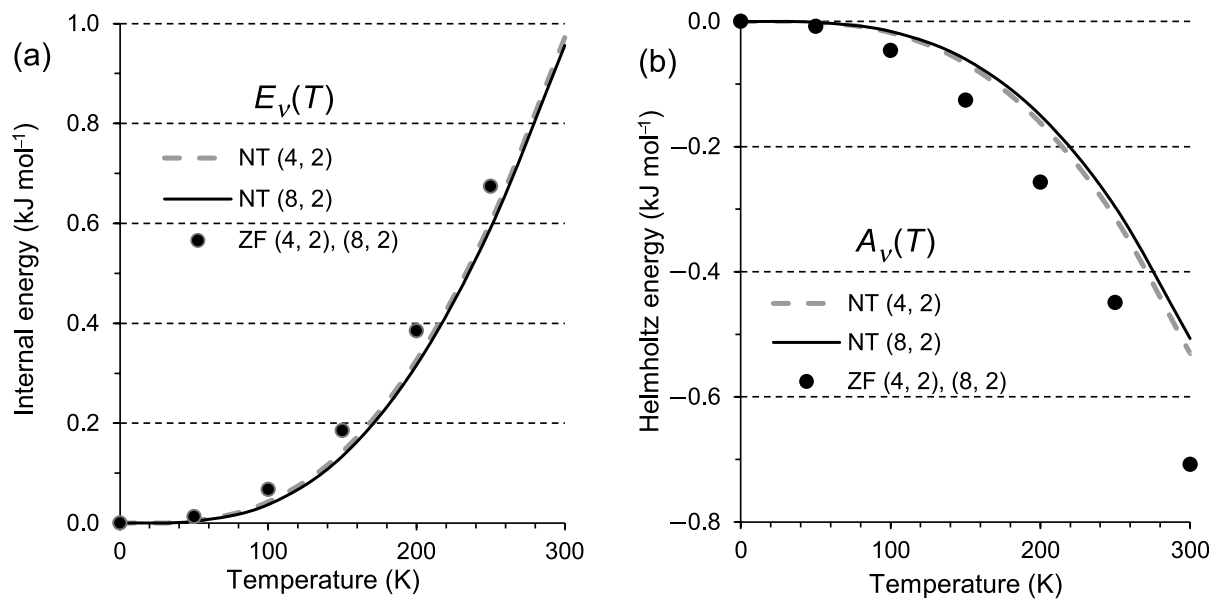

Fig. 5. Temperature dependence of thermal contributions to internal energy (a) and Helmholtz energy (b) for chiral SWCNTs. NT $(4,2)$ (blue online): $D=4.24 \AA,|\mathrm{T}|=22.34 \AA$, $E_{0}=16.42 \mathrm{~kJ} \mathrm{~mol}^{-1}, E_{0}^{\mathrm{ZF}}=$ $16.88 \mathrm{~kJ} \mathrm{~mol}^{-1}$; NT $(8,2)$ : $D=7.19 \AA,|\mathrm{T}|=12.93 \AA$, $E_{0}=16.88 \mathrm{~kJ} \mathrm{~mol}^{-1}, E_{0}^{\mathrm{ZF}}=$ $16.88 \mathrm{~kJ} \mathrm{~mol}^{-1}$.

\section{2. $\mathrm{TiO}_{2}, \mathrm{TiS}_{2}, \mathrm{ZrS}_{2}$, and $\mathrm{V}_{2} \mathrm{O}_{5}$-based nanotubes}

The symmetry group of $\mathrm{TiO}_{2}, \mathrm{TiS}_{2}$ and $\mathrm{ZrS}_{2}$-based SWNT with hexagonal morphology depends on the chirality type. The parent hexagonal layers have layer group $72(P \overline{3} / \mathrm{ml})$ which is the subgroup of space group $164(P \overline{3} / \mathrm{ml})$ - the symmetry group of the most stable bulk TiS ${ }_{2}$ and $\mathrm{ZrS}_{2}$ phases [28]. As follows from Refs. [27, 28], after the rolling procedure the symmetry of the achiral $(n, 0),(n, n)$ and chi- ral $(n, m)$ NTs is described by line groups $L(2 n)_{n} / m$, $L(2 n)_{n} m c$, and $L(N)_{S}$ belonging to families 4 (point symmetry $\left.C_{2 n h}\right), 8$ (point symmetry $C_{2 n v}$ ) and 1 (point symmetry $\mathrm{C}_{N}$ ), respectively. The models for the zigzag and armchair NTs with hexagonal morphology are presented in Figs. 6(a) and (b). In the case of the hexagonal layers the direct and reciprocal lattices are the same as those considered above for graphene. So, the ZF approach developed for the carbon nanotubes can be applied in the same 

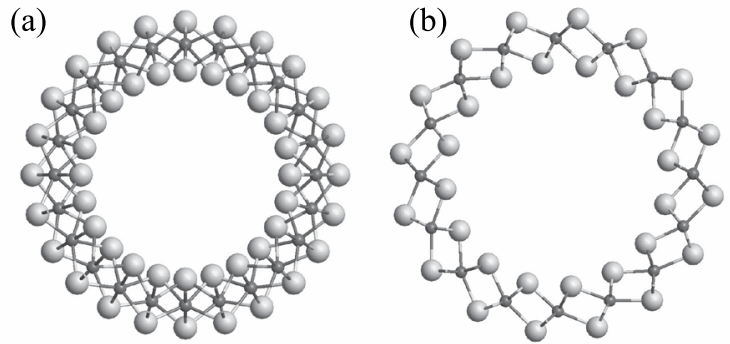

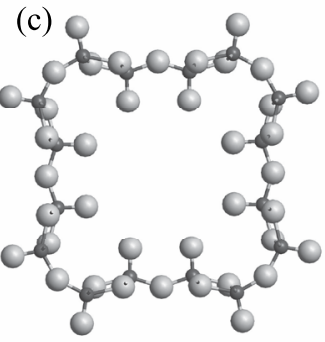

Fig. 6. Optimized structure of (a) zigzag $(12,0) \mathrm{ZrS}_{2}$, (b) armchair $(8,8) \mathrm{ZrS}_{2}$, and (c) $(4,0) \mathrm{V}_{2} \mathrm{O}_{5}$ single-wall nanotubes. Metal atoms are small dark balls (blue and green online), $\mathrm{O}$ or $\mathrm{S}$ atoms are large light balls (yellow and pink). way to the metal oxide and sulfide nanotubes with hexagonal morphology.

A layer group of the $\alpha-\mathrm{V}_{2} \mathrm{O}_{5}$ layer is Pmmn [29, 30]. Only $(n, 0)$ and $(0, m)$ chiralities of SWNTs are compatible with rectangular symmetry of the $\mathrm{V}_{2} \mathrm{O}_{5}$ layer. The SWNTs of both chirality types belong to the 11th family of line symmetry groups $(\mathrm{Ln} / \mathrm{mmm}$ or $L \overline{2} n 2 m$ for even or odd $n$, respectively). Only $(n, 0)$ SWNTs (with $n=4$ and 6) have been considered here because of their greater stability compared to $(0, m)$ NTs [30]. The cross-sectional view of $\mathrm{V}_{2} \mathrm{O}_{5}(4,0)$ SWNT is shown in Fig. 6(c). The primitive vectors $\left[\mathbf{b}_{1}, \mathbf{b}_{2}\right]$ of the reciprocal lattice are mutually orthogonal in the case of the rectangular lattice. In the fully $\mathbf{K}_{1}$-extended representation of $(n, 0)$ SWNTs the cutting lines are parallel to the vector $\mathbf{b}_{2}$ and extended in the $\mathbf{K}_{1}$ direction which is collinear with $\mathbf{b}_{1}$.
The usefulness of the ZF approach is displayed more clearly when we compare the thermodynamic properties of systems with different chemical composition. The temperature dependences of thermal contributions to the internal and Helmholtz free energy calculated for some non-carbon nanotubes directly and using the ZF approach are given in Fig. 7. The comparison of the internal energy dependence shows a good overall agreement between the NT and ZF results. At $300 \mathrm{~K}$ the maximal deviation of about $0.2 \mathrm{~kJ}$ per mole of atoms is found in the case of $\mathrm{TiS}_{2}$ NTs. The corresponding difference for the Helmholtz free energy is three times greater: about $0.6 \mathrm{~kJ}$ per mole of atoms. The best agreement between the $\mathrm{ZF}$ and NT direct values is found for the $\mathrm{TiO}_{2}$ NTs. At $300 \mathrm{~K}$ the $\mathrm{ZF}$ approach reproduces the internal energy of $\mathrm{TiO}_{2} \mathrm{NTs}$ with the accuracy greater than $0.05 \mathrm{~kJ}$ per mole of atoms.
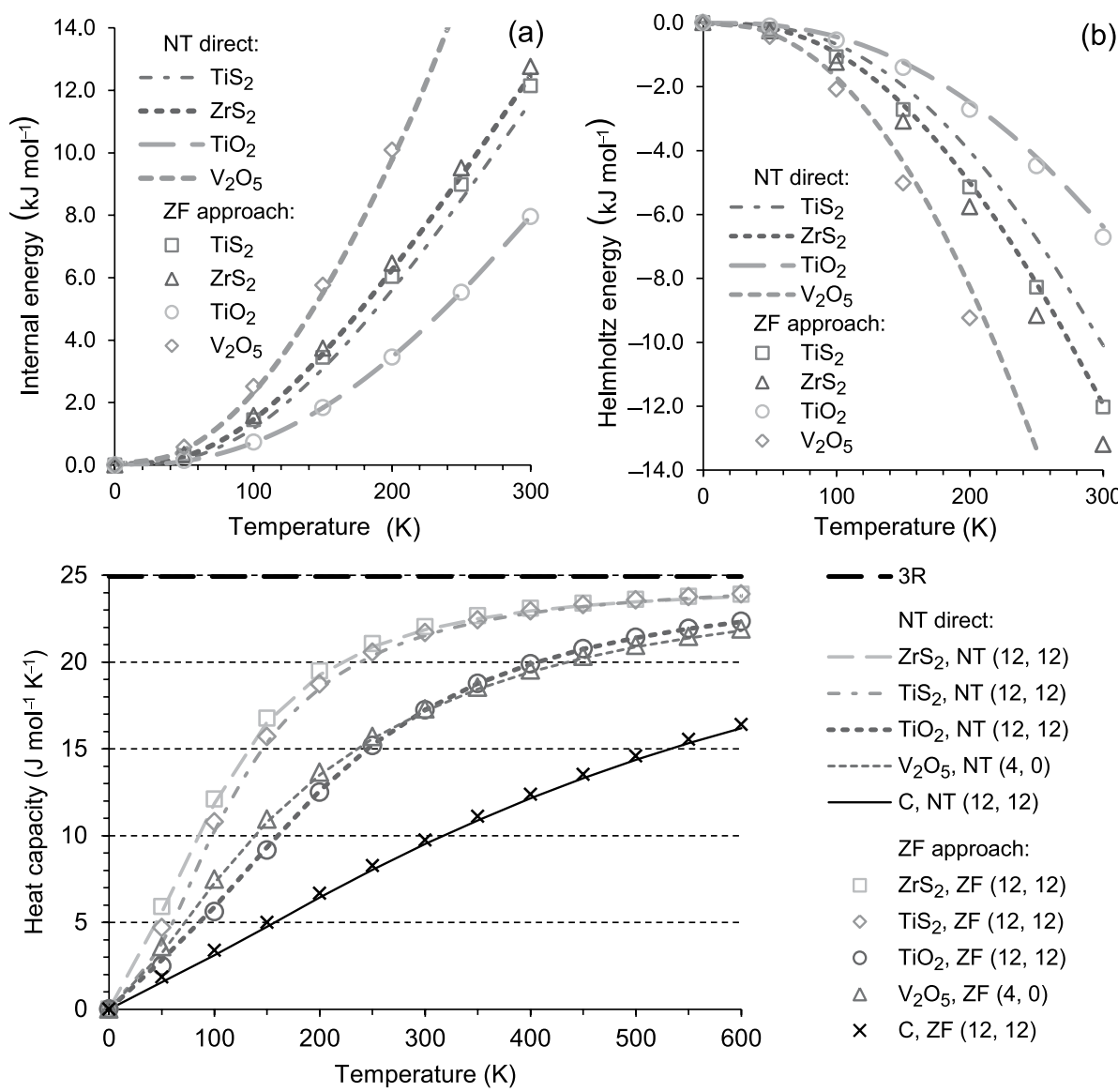

(b)

Fig. 7. Temperature dependence of thermal contributions (per formula unit) to internal energy (a) and Helmholtz energy (b) for $(8,8) \mathrm{TiO}_{2}$ (green online), $\mathrm{TiS}_{2}$ (violet) and $\mathrm{ZrS}_{2}$-based NTs (dark red online) and for $(4,0) \mathrm{V}_{2} \mathrm{O}_{5}$-based NT (blue).
$-3 R$

NT direct:

$-\mathrm{ZrS}_{2}, \mathrm{NT}(12,12)$

- - $\mathrm{TiS}_{2}$, NT $(12,12)$

$\ldots \mathrm{TiO}_{2}, \mathrm{NT}(12,12)$

----- $\mathrm{V}_{2} \mathrm{O}_{5}, \mathrm{NT}(4,0)$

C, NT $(12,12)$

ZF approach:

$\mathrm{ZrS}_{2}, \mathrm{ZF}(12,12)$

$\mathrm{TiS}_{2}, \mathrm{ZF}(12,12)$

$\mathrm{TiO}_{2}, \mathrm{ZF}(12,12)$

$\triangle \mathrm{V}_{2} \mathrm{O}_{5}, \mathrm{ZF}(4,0)$

$\times \quad \mathrm{C}, \mathrm{ZF}(12,12)$
Fig. 8. Temperature dependence of heat capacity (per 1 mole of atoms) of $\mathrm{TiO}_{2}$ (dark red online), $\mathrm{TiS}_{2}$ (blue), $\mathrm{ZrS}_{2}$ (green), $\mathrm{V}_{2} \mathrm{O}_{5}$-based (violet) and graphene-based SWNTs calculated directly and with zone-folding approximation. The DulongPetit limit is shown on the top by a straight dashed line. 
Figure 8 shows the temperature dependences of the heat capacity $C_{V}$ calculated here for $(12,12)$ SWNTs with hexagonal morphology and for $(4,0)$ SWNT rolled up from the single layer of the $\alpha$-phase of $\mathrm{V}_{2} \mathrm{O}_{5}$. The results obtained by both the direct NT phonon calculation and the ZF approach are given. Graphs in Fig. 8 show that the ZF approach can correctly predict the distinctions in the heat capacity exhibited by the indicated systems within the temperature interval from 0 to $600 \mathrm{~K}$. Moreover, it can be seen that the ZF approach can reproduce the peculiar features of the temperature dependences even in the cases of close $C_{V}$ values.

\section{Conclusions}

The opportunities of first-principles methods in the calculation of NT thermodynamic properties are currently limited to nanotubes with relatively small diameters which are considerably lower than those experimentally observed. This is due to the fact that computational expenses rise quickly with the increase of NT diameter. The present study shows that this problem can, at least partially, be solved by using the zone-folding approach. We have performed the first-principles calculations for a wide range of carbon and non-carbon (transition metal oxide and sulfide) nanotubes and we have found that the values of heat capacity and internal energy obtained by direct SWNT and layer zone-folding calculations practically coincide at low temperatures and remain very close up to $600 \mathrm{~K}$. Although the agreement is worse for the Helmholtz free energy and entropy, the zone-folding approach is still acceptable for the approximate estimation of these thermodynamic functions with the accuracy of about $0.5 \mathrm{~kJ}$ per mole of atoms in the abovementioned temperature range.

\section{Acknowledgements}

The authors acknowledge the financial support from the Saint Petersburg State University (grants 12.38.273.2014 and 12.50.1566.2013) and assistance of the University Computer Center in the accomplishment of high-performance computations. RAE and AVB are also grateful for funding of $\mathrm{TiO}_{2}$ SWNT computations provided by the Russian Foundation for Basic Research (Grant No. 14-03-00107-a).

\section{References}

[1] R.A. Evarestov, Theoretical Modeling of Inorganic Nanostructures. Symmetry and Ab-Initio Calculations of Nanolayers, Nanotubes and Nanowires, Springer Series in NanoScience and Technology
(Springer, Berlin, Heidelberg, 2015), http://dx.doi. org/10.1007/978-3-662-44581-5

[2] Ge.G. Samsonidze, R. Saito, A. Jorio, M.A. Pimenta, A.G. Souza Filho, A. Grüneis, G. Dresselhaus, and M.S. Dresselhaus, The concept of cutting lines in carbon nanotube science, I. Nanosci. Nanotech. 3, 431-458 (2003), http://dx.doi.org/10.1166/ inn.2003.231

[3] O. Dubay and G. Kresse, Accurate density functional calculations for the phonon dispersion relations of graphite layer and carbon nanotubes, Phys. Rev. B 67, 035401 (2003), http://dx.doi. org/10.1103/PhysRevB.67.035401

[4] T.B. Boykin and G. Klimeck, Practical application of zone-folding concepts in tight-binding calculations, Phys. Rev. B 71, 115215 (2005), http:// dx.doi.org/10.1103/PhysRevB.71.115215

[5] R. Saito, K. Sato, Y. Oyama, J. Jiang, Ge.G. Samsonidze, G. Dresselhaus, and M.S. Dresselhaus, Cutting lines near the Fermi energy of single-wall carbon nanotubes, Phys. Rev. B 72, 153413 (2005), http://dx.doi.org/10.1103/PhysRevB.72.153413

[6] J-C. Charlier, X. Blase, and S. Roche, Electronic and transport properties of nanotubes, Rev. Mod. Phys. 79, 677-732 (2007), http://dx.doi. org/10.1103/RevModPhys.79.677

[7] R. Sahoo and R.R. Mishra, Phonon dispersion for armchair and zigzag carbon nanotubes, Graphene 3,14-19(2014), http://dx.doi.org/10.4236/graphene. 2014.32003

[8] D. Sanchez-Portal and E. Hernandez, Vibrational properties of single-wall nanotubes and monolayers of hexagonal BN, Phys. Rev. B 66, 235415 (2002), http://dx.doi.org/10.1103/PhysRevB.66.235415

[9] L. Wirtz, A. Rubio, R.A. de la Concha, and A. Loiseau, $A b$ initio calculations of the lattice dynamics of boron nitride nanotubes, Phys. Rev. B 68, 045425 (2003), http://dx.doi.org/10.1103/PhysRevB.68.045425

[10]P.G. Radaelli, Symmetry in Crystallography: Understanding the International Tables, IUCr Texts on Crystallography, Vol. 17 (Oxford University Press, Oxford, 2011), http://dx.doi.org/10.1093/ acprof:oso/9780199550654.001.0001

[11] K. Kunc and R.M. Martin, Ab initio force constants of GaAs: A new approach to calculation of phonons and dielectric properties, Phys. Rev. Lett. 48, 406-409 (1982), http://dx.doi.org/10.1103/ PhysRevLett.48.406

[12]F. Pascale, C.M. Zicovich-Wilson, F. Lopez, B. Civalleri, R. Orlando, and R. Dovesi, The calculation of the vibrational frequencies of crystalline compounds and its implementation in the CRYSTAL code, J. Comput. Chem. 25, 888897 (2004), http://dx.doi.org/10.1002/jcc.20019

[13] D.C. Wallace, Thermodynamics of Crystals (Dover, New York, 1998).

[14] C. Adamo and V. Barone, Toward reliable density functional methods without adjustable parameters: 
The PBE0 model, J. Chem. Phys. 110, 6158-6170 (1999), http://dx.doi.org/10.1063/1.478522

[15] R. Dovesi, R. Orlando, A. Erba, C.M. ZicovichWilson, B. Civalleri, S. Casassa, L. Maschio, M. Ferrabone, M. De La Pierre, Ph. D’Arco, Y. Noël, M. Causà, M. Rérat, and B. Kirtman, CRYSTAL14: A program for the $a b$ initio investigation of crystalline solids, Int. J. Quant. Chem. 114, 1287-1317 (2014), http://dx.doi.org/10.1002/qua.24658

[16] R. Dovesi, V.R. Saunders, C. Roetti, R. Orlando, C.M. Zicovich-Wilson, F. Pascale, B. Civalleri, K. Doll, N.M. Harrison, I.J. Bush, Ph. D’Arco, M. Llunell, M. Causà, and Y. Noël, CRYSTAL14 User's Manual (University of Torino, Torino, 2014), http://www.crystal.unito.it/index.php

[17] M.F. Peintinger, D.V. Oliveira, and T. Bredow, Consistent Gaussian basis sets of triple-zeta valence with polarization quality for solid-state calculations, J. Comput. Chem. 34, 451-459 (2013), http://dx.doi.org/10.1002/jcc.23153

[18] L.F. Pacios and P.A. Christiansen, $A b$ initio relativistic effective potentials with spin-orbit operators. I. Li through Ar, J. Chem. Phys. 82, 2664-2671 (1985), http://dx.doi.org/10.1063/1.448263

[19] M.M. Hurley, L.F. Pacios, P.A. Christiansen, R.B. Ross, and W.C. Ermler, $A b$ initio relativistic effective potentials with spin-orbit operators. II. K through Kr, J. Chem. Phys. 84, 6840-6853 (1986), http://dx.doi.org/10.1063/1.450689

[20] L.A. LaJohn, P.A. Christiansen, R.B. Ross, T. Atashroo, and W.C. Ermler, Ab initio relativistic effective potentials with spin-orbit operators. III. Rb through Xe, J. Chem. Phys. 87, 2812-2824 (1987), http://dx.doi.org/10.1063/1.453069

[21] A.V. Bandura and R.A. Evarestov, Structure and stability of $\mathrm{SnS}_{2}$-based single- and multi-wall nanotubes, Surf. Sci. 641, 6-15 (2015), http://dx.doi. org/10.1016/j.susc.2015.04.027

[22] H.J. Monkhorst and J.D. Pack, Special points for Brillouin-zone integrations, Phys. Rev. B 13,
5188-5192 (1976), http://dx.doi.org/10.1103/ PhysRevB.13.5188

[23] C.M. Zicovich-Wilson, F. Pascale, C. Roetti, V.R. Saunders, R. Orlando, and R.J. Dovesi, Calculation of vibration frequencies of alphaquartz: the effect of Hamiltonian and basis set, J. Comput. Chem. 25, 1873-1881 (2004), http:// dx.doi.org/10.1002/jcc.20120

[24] M. Damnjanović and I. Milošević, Line Groups in Physics. Theory and Applications to Nanotubes and Polymers, Lecture Notes in Physics, Vol. 801 (Springer, Berlin, 2010), http://dx.doi. org/10.1007/978-3-642-11172-3

[25] S. Reich, C. Thomsen, and J. Maultzsch, Carbon Nanotubes: Basic Concepts and Physical Properties (Wiley, Weinheim, 2004), http://dx.doi. org/10.1002/9783527618040

[26]M.S. Dresselhaus, G. Dresselhaus, and A. Jorio, Group Theory. Application to the Physics of Condensed Matter (Springer, Berlin, 2008), http://dx.doi. org/10.1007/978-3-540-32899-8

[27] M. Damnjanović, B. Nicolić, and I. Milošević, Symmetry of nanotubes rolled up from arbitrary two-dimensional lattices along an arbitrary chiral vector, Phys. Rev. B 75, 033403 (2007), http:// dx.doi.org/10.1103/PhysRevB.75.033403

[28] A.V. Bandura and R.A. Evarestov, $\mathrm{TiS}_{2}$ and $\mathrm{ZrS}_{2}$ single- and double-wall nanotubes: first-principles study, J. Comp. Chem. 35, 395-405 (2014), http:// dx.doi.org/10.1002/jcc.23508

[29] V. Shklover, T. Haibach, F. Ried, R. Nesper, and P. Novák, Crystal structure of the product of $\mathrm{Mg}^{2+}$ insertion into $\mathrm{V}_{2} \mathrm{O}_{5}$ single crystals, I. Solid State Chem. 123, 317-323 (1996), http://dx.doi. org/10.1006/jssc.1996.0186

[30] V.V. Porsev, A.V. Bandura, and R.A. Evarestov, Ab initio modeling of single wall nanotubes folded from $\alpha$ and $\gamma-\mathrm{V}_{2} \mathrm{O}_{5}$ monolayers: structural, electronic and vibrational properties, CrystEngComm 17, 3277-3285 (2015), http://dx.doi.org/10.1039/c5ce00144g

\title{
TERMODINAMINĖS NANOVAMZDELIŲ SAVYBĖS: ZONŲ PERLENKIMO METODAS
}

\author{
R.A. Evarestov, A.V. Bandura, V.V. Porsev \\ Sankt Peterburgo valstybinis universitetas, Rusija
}

